\title{
Fabrication and modeling of multimode fiber lenses
}

\author{
Klaus Bescherer, ${ }^{1}$ Dorit Munzke, ${ }^{2}$ Oliver Reich, ${ }^{2}$ and Hans-Peter Loock ${ }^{1, *}$ \\ ${ }^{1}$ Department of Chemistry, Queen's University, Kingston, Ontario K7L 3N6, Canada \\ 2innoFSPEC, Physical Chemistry, University of Potsdam, Potsdam D-14476, Germany \\ *Corresponding author: hploock@chem.queensu.ca
}

Received 18 September 2012; revised 5 December 2012; accepted 6 December 2012; posted 6 December 2012 (Doc. ID 176449); published 16 January 2013

\begin{abstract}
We report on the fabrication, modeling, and experimental verification of the emission of fiber lenses fabricated on multimode fibers in different media. Concave fiber lenses with a radius of $150 \mu \mathrm{m}$ were fabricated onto a multimode silica fiber $(100 \mu \mathrm{m}$ core) by grinding and polishing against a ruby sphere template. In our theoretical model we assume that the fiber guides light from a Lambertian light source and that the emission cone is governed solely by the range of permitted emission angles. We investigate concave and convex lenses at $532 \mathrm{~nm}$ with different radii and in a variety of surrounding media from air $\left(n_{0}=1.00\right)$ to sapphire $\left(n_{0}=1.77\right)$. It was found that noticeable focusing or defocusing effects of a silica fiber lens in ethanol $\left(n_{0}=1.36\right)$ and dimethyl sulfoxide (DMSO) $\left(n_{0}=1.48\right)$ are only observed when the fiber lens radius was less than the fiber diameter. () 2013 Optical Society of America

OCIS codes: $\quad 060.2310,080.3630,080.1753$.
\end{abstract}

\section{Introduction}

Many applications in spectroscopy, scanning probe microscopy, sensing, and detection require that light from a power delivery fiber is focused onto a sample. Conversely, light from a light source such as a lightemitting diode or a laser frequently needs to be coupled into a multimode delivery fiber. Fiber microlenses may be used to achieve high coupling efficiencies and can be fabricated either directly onto the end of multimode fibers or onto a spacer.

In recent decades many different lens types were fabricated onto the end of a fiber-optic waveguide, such as hemispherical convex lenses using thermal melting [1-5] or axicon lenses, which may be made either by grinding and polishing [6] , by laser machining [7], or by ion beam milling []. Focused ion beams were used to fabricate a diffractive optical element lens [9] and a Fresnel lens [10] on the end face of a singlemode fiber, and Fresnel lenses could also be fabricated by etching [11]. Most lenses were convex, but concave lenses were also made either by laser

1559-128X/13/040B40-06 $\$ 15.00 / 0$

(C) 2013 Optical Society of America machining [12] or using a heated spherical template that was embossed into the fiber end [13]. Inverse, (concave) conical lenses that convert a Gaussian beam into a flat-top profile have also been made by etching [14].

Beside micromachining at the end of the silica fiber, there exist a large number of microfabrication techniques with which lenses may be made from polymers and affixed to the end of a waveguide, such as using the light force to attach polystyrene glass beads and convex lenses [15], compression molding [16], laser polymerization [17], microjet deposition [18], and methods exploiting the surface tension of $\overline{\mathrm{UV}}$-photocurable polymers [19].

Many previous articles quantify the focusing or collimation characteristics of these lenses, and theoretical models have been developed to describe and predict fiber lens properties. Besides the common finite-difference time-domain methods, we believe that geometric (ray) optics remains a useful tool to calculate the lens properties of fiber lenses when the fiber core diameter is substantially larger than the wavelength of light $[\underline{1}, \underline{3}, 20]$.

As part of an attempt to develop fiber-optic probes for the on-line analysis of lubricants [21], we recently 
conducted a study of fiber-optic fluorescence excitation and detection of emission in the presence of primary and secondary absorption [22]. The previous work was inspired by the simple Lambertian model that He and Cuomo [23] developed for the description of the emission from a multimode silica fiber and the excellent agreement they found with experimental observations. Briefly, the model assumes that every point on the planar fiber core surface emits light into all directions within the critical acceptance angle of the fiber. The intensity at any point in the irradiated volume is then obtained by adding the "rays" converging to this point and weighing with the appropriate divergence factor to conserve energy.

While the equations that $\mathrm{He}$ and Cuomo derived are no longer valid for curved fiber surfaces, a similar model may nevertheless be used. In this report we present results from a ray optics calculation that provides a quantitative description of the observed intensity distribution resulting from a Lambertian light source coupled into a multimode fiber. We also provide instructions on how a concave fiber lens may be fabricated onto the end of a $100 / 140 \mu \mathrm{m}$ multimode silica fiber.

\section{Lens Fabrication}

The concave lens at one fiber end was fabricated by polishing the fiber end with a $300 \mu \mathrm{m}$ diameter ruby sphere (http://www.smallparts.com; the item is no longer available). The ruby sphere was affixed to a stainless steel fiber ferrule holder (Precision Fiber Products, MM-CON2003-SS-1260), which was firmly held in place. The multimode fiber $(100 \mu \mathrm{m}$ core and $140 \mu \mathrm{m}$ cladding diameter) was also held in a ferrule, which was clamped into an aluminium cylinder. That assembly was rotated around the fiber axis using an oscillatory motor (from an electric toothbrush). Lapping paper (aluminium oxide $0.3 \mu \mathrm{m}$, Fiber Optic Center Inc.) was held in between the ruby sphere holder and the fiber ferrule, and the ferrule was gently pressed against the lapping paper, which conformed to the shape of the template. Water was not used in this polishing process. A scanning electron microscope (SEM) picture of a concave fiber lens is shown in Fig. 1(a). Even at low magnification a small dip in the center of the ferrule can be seen. An optical micrograph of the ruby sphere template [Fig. 1(b)] shows the spherical protrusion by the sphere.

\section{Theoretical Model}

We assume that each of the points of the surface of the lensed fiber emits rays within an acceptance angle that depends not only on the numerical aperture (NA) of the fiber and the refractive index of the solution, $n_{0}$, but also on the curvature of the fiber surface, $\arcsin (r / R)$. Here, $R$ is the radius of the spherical lens, $r_{\text {core }}$ is the fiber core radius, and $r$ is the distance of the emitting point on the lens from the fiber axis. The intensity, $I$, at a point $\{z, x\}$ in the solution volume can be obtained by collecting the intensity of the rays that are emitted at angles $\alpha$ from the fiber end and at a radial distance $r$ from the fiber axis

$$
I(x, z)=\int_{-r_{\text {core }}}^{+r_{\text {core }}} I(\alpha, r) w\left(\beta_{1}, \alpha, \beta_{2}\right) \mathrm{d} r
$$

The angles

$$
\alpha=\arctan \left(\frac{x-r}{z}\right)
$$

are formed between the beam axis and the ray originating from the fiber surface at $r$ to point $\{z, x\}$. This angle has to fall between the two limiting "critical" angles $\beta_{1}$ and $\beta_{2}$. When this condition is fulfilled, the windowing function $w\left(\beta_{1}, \alpha, \beta_{2}\right)=1$, and it is zero otherwise. In the calculations below we chose the intensity distribution function $I(\alpha, r)=1$ for a Lambertian fiber source, but one may select it to be any other function depending on the properties of the multimode fiber and the light source coupling light into the fiber. Another interesting case is the perfectly collimated source in which $I(\alpha, r)=1$ for $\alpha=0$ and $I(\alpha, r)=0$ otherwise.

In practice we use a discretized form of the integral in Eq. (1) and simply count the number of rays converging to the point $\{z, x\}$ :
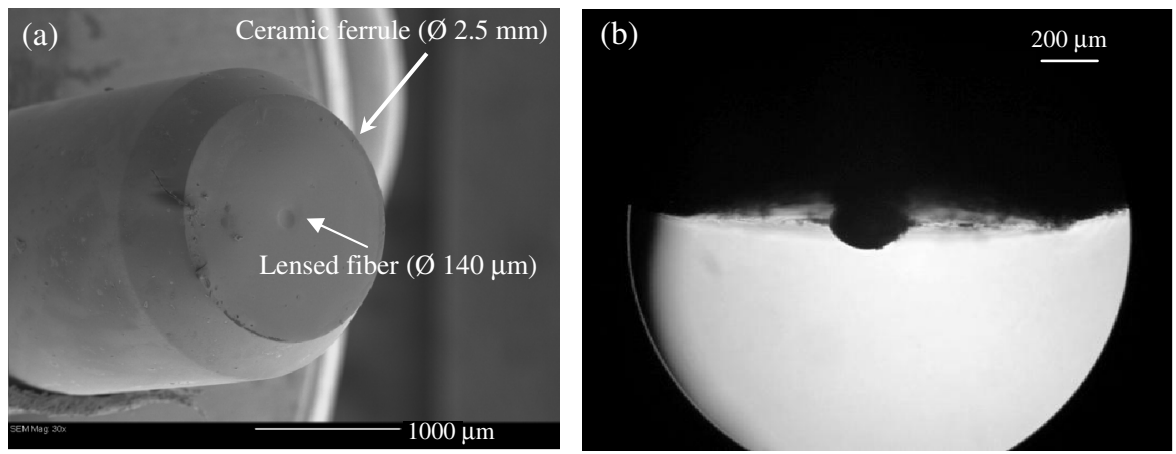

Fig. 1. (a) SEM picture of the concave lens at the fiber ferrule. (b) Micrograph at 10× magnification of the $R=150 \mu \mathrm{m}$ ruby microsphere inserted in a stainless steel fiber ferrule. This template was used to fabricate a concave fiber lens. 
$I(x, z)$
$=\sum_{r=-r_{0}}^{r_{0}} \begin{gathered}I(\alpha, r) \\ 0\end{gathered} \quad$ if $\beta_{1}(r)>\alpha(r, x, z)$ or $\alpha(r, x, z)>\beta_{2}(r)$.

For a planar fiber surface these angles would be simply $\beta_{1}=-\beta_{2}=\arcsin \left(\mathrm{NA} / n_{0}\right)$. For a curved surface, the relation is more complicated, and a ray diagram is given in Fig. 2.

From the figure one can readily verify that, for a convex lens, the respective angles depend on the refractive indices of core $\left(n_{\text {core }}\right)$ and cladding $\left(n_{\text {clad }}\right)$ :

$$
\begin{aligned}
\gamma & =\arcsin \left(\frac{r}{R}\right), \\
\theta_{c} & =\arcsin \left(\frac{n_{\text {clad }}}{n_{\text {core }}}\right), \quad n_{0} \sin \phi_{s}=n_{\text {core }} \sin \phi_{f} .
\end{aligned}
$$
of

The critical angle $\beta_{1}$ is then obtained by insertion

$$
\phi_{f}=\frac{\pi}{2}-\theta_{c}+\gamma, \quad \beta_{1}=\phi_{s}-\gamma,
$$

and after simplification, one obtains

$$
\begin{aligned}
\beta_{1}= & -\arcsin \left(\frac{r}{R}\right) \\
& +\arcsin \left[\frac{n_{\text {core }}}{n_{0}} \cos \left(\arcsin \left(\frac{r}{R}\right)-\arcsin \left(\frac{n_{\text {clad }}}{n_{\text {core }}}\right)\right)\right] \\
= & -\arcsin \left(\frac{r}{R}\right)+\arcsin \left[\frac{\mathrm{NA} \sqrt{R^{2}-r^{2}}+r n_{\text {clad }}}{R n_{0}}\right] .
\end{aligned}
$$

Similarly, the other limiting angle $\beta_{2}$ for rays arriving at the interface at an angle of $-\theta_{c}$ is derived with

$$
\phi_{f}=\frac{\pi}{2}-\theta_{c}-\gamma, \quad \beta_{2}=\gamma+\phi_{s},
$$

as

$$
\beta_{2}=\arcsin \left(\frac{r}{R}\right)+\arcsin \left[\frac{\mathrm{NA} \sqrt{R^{2}-r^{2}}-r n_{\mathrm{clad}}}{R n_{0}}\right]
$$

The example calculated here for a positive curvature is easily adapted to a negative curvature (a concave lens) using $R<0$ and switching $\beta_{1}$ and $\beta_{2}$. Note that, with these definitions, $\beta_{1}$ and $\beta_{2}$ are both positive for positive $R$ and are both negative for negative $R$. Also, the signs for angles will have to be watched when the curvature of the lens is very large (i.e., at small $R$ ) and $\theta_{c}+\gamma>\pi / 2$.

Three more transformations were used. First, we generated curved surfaces at the fiber interface. All pixels $\{z, x\}$ were moved within each row according to

$$
\begin{array}{ll}
z \rightarrow z-R-\sqrt{R^{2}-r^{2}} & \text { if } R<0 \text { and } r<r_{\text {core }}, \\
z \rightarrow z-R-\sqrt{R^{2}-r_{\text {core }}^{2}} & \text { if } R<0 \text { and } r>r_{\text {core }}, \\
z \rightarrow z+\sqrt{R^{2}-r^{2}}-\sqrt{R^{2}-r_{\text {core }}^{2}} & \text { if } R>0 \text { and } r<r_{\text {core }}, \\
z \rightarrow z & \text { if } R>0 \text { and } r>r_{\text {core }} .
\end{array}
$$

These transformed images were used later to calculate the coupling efficiencies between the emitting fiber and an "ideal" receiving fiber with a flat fiber surface and the same dimensions and NA. Second, the intensity decay with distance from the fiber surface was forced to conserve energy; i.e., in the images of Fig. 3 each column of pixels was recalibrated to have the same total intensity. This condition somewhat reduces the effect of the ripples seen at short distance from the fiber end, which are a result of discretization when calculating the angles.

Finally an Abel transformation was used to generate images that can then be compared directly to those observed through the objective of a microscope.
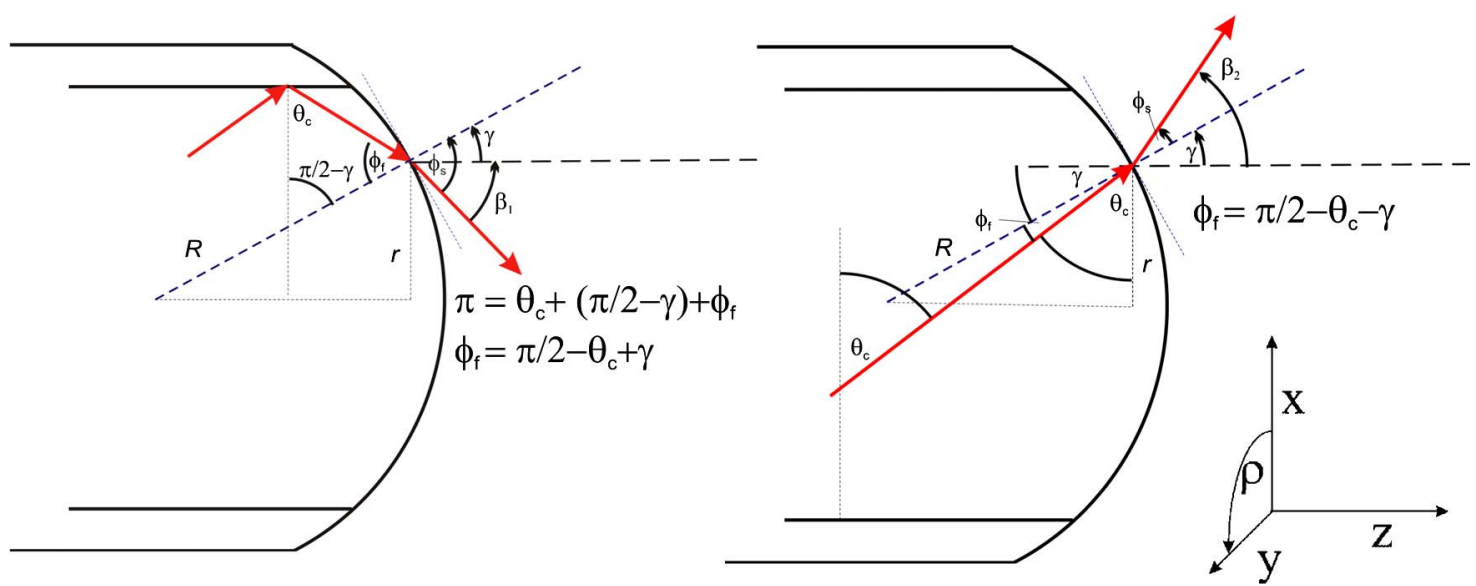

Fig. 2. (Color online) Ray diagrams showing the calculation of the limiting angles (left) $\beta_{1}$ and (right) $\beta_{2}$ as a function of the lens radius, $R$, and the critical angle for wave guiding $\theta_{c}$. 


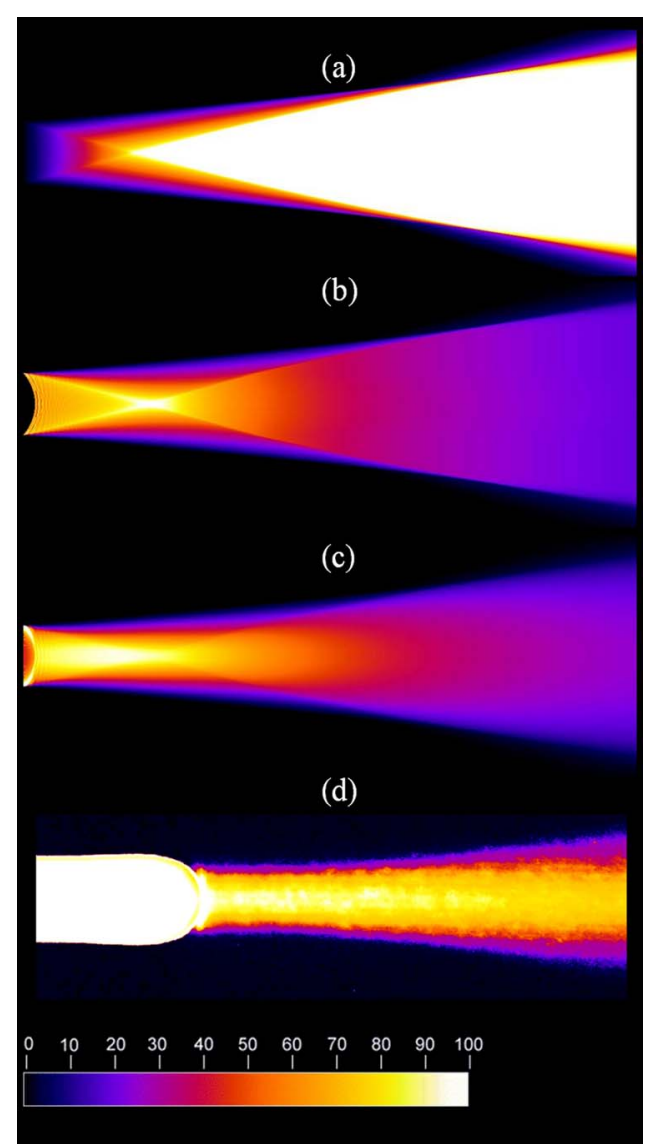

Fig. 3. (Color online) Fiber lens emission cones with a fiber lens radius $R=75 \mu \mathrm{m}$ in water $\left(n_{0}=1.33\right)$. In this and all other images the intensity is color coded to produce a false color image. The simulation window is $0.4 \mathrm{~mm}$ high and $1.0 \mathrm{~mm}$ long. The fiber core diameter is $100 \mu \mathrm{m}, \mathrm{NA}=0.22, n_{\text {clad }}=1.433$. (a) As calculated from Eq. (3), (b) after normalization and translation to include a lens using Eq. (9), and (c) after Abel transformation using Eq. (10). Ripples at short distance from the fiber lens are a discretization artifact of the simulation. (d) Experimental data on the same scale recorded using a microscope and adapted from [4]].

This involves, first, to produce a cylindrically symmetric 3D distribution of excitation intensities by rotation of the $2 \mathrm{D}$ distribution of Fig. $\underline{3}$ around the center axis, $I(r, \rho, z)$, where $\rho$ is the rotation angle (see also Fig. 2). This is followed by a projection on the detection plane (Fig. 3) [22]:

$$
\begin{aligned}
I(x, z) & =2 \int_{x}^{\infty} \int_{0}^{2 \pi} I(r, \rho, z) \frac{r}{\sqrt{r^{2}-x^{2}}} \mathrm{~d} \rho \mathrm{d} r \\
& =4 \pi \int_{x}^{\infty} I(r, z) \frac{r}{\sqrt{r^{2}-x^{2}}} \mathrm{~d} r .
\end{aligned}
$$

The results of these transformations on a concave lens with $R=-75 \mu \mathrm{m}$ are shown in Fig. 3. Figure 4 shows the emission of a concave lens with a curvature of $R=-150 \mu \mathrm{m}$ in air, ethanol, dimethyl sulfoxide (DMSO), and sapphire. As may be expected, the concave lens is diverging in air and ethanol and converging in DMSO and other media with $n_{0}>n_{\text {core }}$.

From the 2D representation the maximal coupling efficiency between the lensed fiber and a flat fiber with otherwise identical dimensions and NA was calculated as a function of distance between the lens and the receiving fiber. Figure 5 shows the coupling efficiency for concave lenses with radii up to $R=$ $-60 \mu \mathrm{m}$ and convex lenses with radii up to $R=+60 \mu \mathrm{m}$. The coupling efficiency has been calculated in media with refractive indices of $n_{0}=1.0$, $1.33,1.48$, and 1.77 , corresponding approximately to air, water, DMSO, and sapphire, respectively. In the calculation we assumed that there are no other optical losses except geometric coupling losses and neglected the considerable effect of scattering and Fresnel reflection at the fiber lens surface.

Comparison of the coupling efficiencies with earlier experimental data is particularly instructive, and it is also convenient since the data reported in Fig. 5 scale linearly with the dimensions of the system; i.e., the coupling efficiency of a $400 \mu \mathrm{m}$ core diameter waveguide over a $4 \mathrm{~mm}$ distance follows the same curve as a the $100 \mu \mathrm{m}$ waveguide over $1 \mathrm{~mm}$ that is presented in the figure.

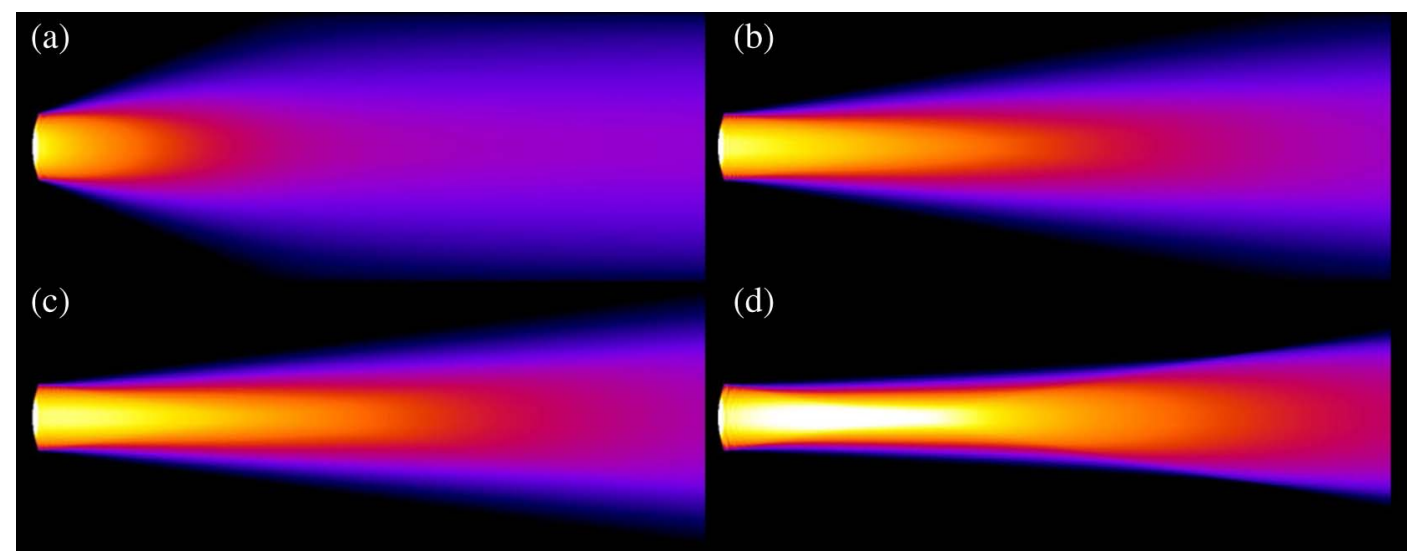

Fig. 4. (Color online) Simulated emission from fiber lens with NA $=0.22, R=-150 \mu \mathrm{m}, r_{\text {core }}=50 \mu \mathrm{m}, n_{\text {core }}=1.433$. The refractive indices of the medium are (a) $n_{0}=1.0$ (air), (b) $n_{0}=1.365$ (ethanol), (c) $n_{0}=1.4785$ (DMSO), and (d) $n_{0}=1.77$ (sapphire). The figure shows the Abel-transformed images. 


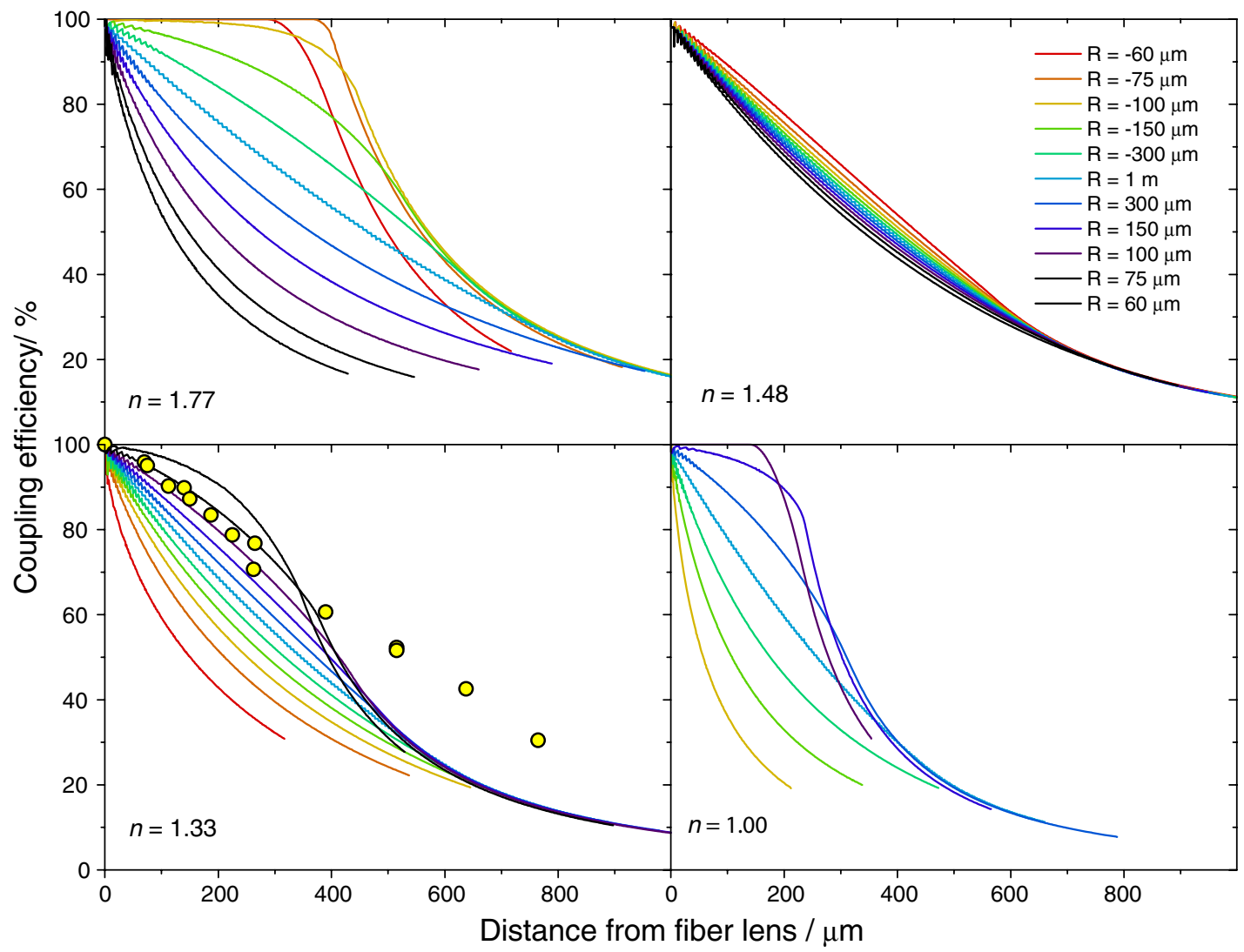

Fig. 5. (Color online) Coupling efficiencies of the fiber lens with radii between $R=-60 \mu \mathrm{m}$ to $+60 \mu \mathrm{m}$ in media with different refractive indices. The fiber core diameter is $100 \mu \mathrm{m}, \mathrm{NA}=0.22, n_{\text {clad }}=1.433$. The circles in the bottom left panel were obtained by reanalysis of the ringdown times recorded from a convex lens $(R \approx+75 \mu \mathrm{m})$ in water [5]. Simulations with a convex or concave lens radius of less than about $80 \mu \mathrm{m}$ could not be performed when $n_{0}=1.0$, since then Eqs. (6) and (8) are no longer valid.

\section{Microscopy}

Light from a $532 \mathrm{~nm}$ cw-laser was diffused using a thin sheet of paper to emulate a Lambertian source and then coupled into a multimode fiber (core

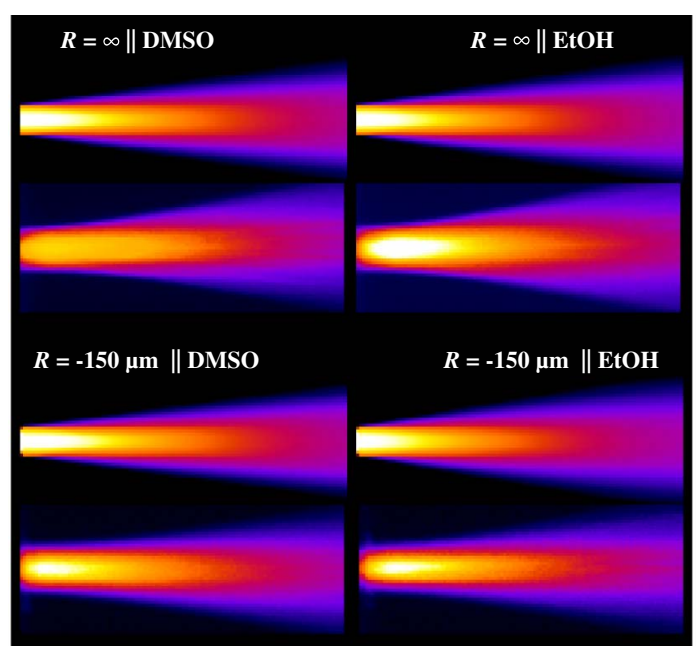

Fig. 6. (Color online) Theoretical and experimental fiber lens emission (top) with a flat fiber end (simulated as $R=1 \mathrm{~m}$ ) and (bottom) with $R=-150 \mu \mathrm{m}$ in (left) pure DMSO and (right) pure ethanol. The window is $0.4 \mathrm{~mm}$ high and $1.0 \mathrm{~mm}$ long. The fiber core diameter is $100 \mu \mathrm{m}, \mathrm{NA}=0.22, n_{\text {clad }}=1.433$. In each group the simulation is shown above the experimental image. diameter $100 \mu \mathrm{m}$, cladding diameter $140 \mu \mathrm{m})$. The fiber was submersed in a petri dish containing solutions of a fluorescent dye (Eosin Y; BASF, Germany; used as supplied) in DMSO (Carl Roth; used without further purification) or basic ethanol (96\%, Carl Roth; used without further purification). The fluorescence emission from flat and concave fiber ends $(R=-150 \mu \mathrm{m})$ was imaged using a microscope (Axio Imager.M2m with EC Epiplan-Neofluar 5× objective, Carl Zeiss MicroImaging, Germany). The images were recorded in 8 bit linear gray scale and processed using Image-J software (W. Rasband, National Institute of Health, USA).

Figure $\underline{6}$ shows microscope images and simulations of the fiber lens emission for a flat $(R=1 \mathrm{~m})$ and concave $(R=-150 \mu \mathrm{m})$ lens in DMSO (left) and ethanol (right). The focusing effect of the concave fiber lens compared to the flat fiber end is negligible in both solvents.

\section{Conclusions}

The results that are shown in Figs. 5 and 6 permit us to draw the following conclusions:

- There is good qualitative and quantitative agreement between the emission cones that are calculated using our analytical model and the experimental microscope images. This indicates that the 
approximation of a Lambertian intensity distribution, $I(\alpha, r)=1$, is adequate. The model is not limited to these distributions, however, and can easily be extended to describe anisotropic emission sources.

- When coupling into water $\left(n_{0}=1.33\right)$ or air $\left(n_{0}=1.00\right)$ the focusing and defocusing effects predicted from the model are quite pronounced, leading, for example, to an increase in coupling efficiency from $69 \%$ for a flat-top fiber at $200 \mu \mathrm{m}$ distance to $76 \%$ for a $R=+150 \mu \mathrm{m}$ lens in water and an increase from $60 \%$ to almost $90 \%$ in air.

- The coupling efficiency of a concave lens with $R=-150 \mu \mathrm{m}$ is about $86 \%$ at $100 \mu \mathrm{m}$ distance and $73 \%$ at a $200 \mu \mathrm{m}$ distance when the surrounding medium is DMSO. The numbers are almost the same for a convex lens (84\% and $70 \%)$ and a flat-top fiber (84\% and $71 \%$ ) so that the effect of such a large fiber lens is small when the medium is nearly indexmatched to silica.

- As expected the concave lenses show a pronounced focusing effect when the surrounding medium has a very high refractive index. As an example, we observe that the concave lens with $R=-150 \mu \mathrm{m}$ can couple into a fiber at $200 \mu \mathrm{m}$ distance with an efficiency of $\sim 92 \%$ compared to about $75 \%$ for a flat-top waveguide at a refractive index of 1.77.

- The agreement of our model with coupling efficiencies recorded previously by ringdown spectroscopy [5] is satisfactory at short distances, but deviations are found at long distances between the fiber ends. This may be attributed to the particular geometry of the previous experiment, which allowed that reflections from a channel wall could reenter the waveguide at long distances.

The authors thank Nick Trefiak for technical assistance in providing the data for Fig. 3(d). H.-P. L. thanks innoFSPEC (Potsdam) and the University of Potsdam for partial support of his stay in Germany. K. B. and H.-P. L. acknowledge GasTOPS Ltd. (Ottawa, Ontario) and the Natural Sciences and Engineering Research Council (NSERC) of Canada for financial support of this work. D. M. and $\mathrm{O}$. R. acknowledge the financial support of the German Federal Ministry of Education and Research (grant 03Z2AN12).

\section{References}

1. D. Kato, "Light coupling from a stripe-geometry GaAs diode laser into an optical fiber with spherical end," J. Appl. Phys. 44, 2756-2758 (1973).

2. U. C. Paek and A. L. Weaver, "Formation of a spherical lens at optical fiber ends with a $\mathrm{CO}_{2}$-laser," Appl. Opt. 14, 294-298 (1975).

3. A. Hokkanen and S. Tammela, "Hemispherically ended optical fiber lenses," Phys. Scr. T69, 159-162 (1997).

4. H. P. Loock, "Ring-down absorption spectroscopy for analytical microdevices," TrAC Trends Anal. Chem. 25, 655-664 (2006).

5. H. Waechter, D. Munzke, A. Jang, and H. P. Loock, "Simultaneous and continuous multiple wavelength absorption spectroscopy on nanoliter volumes based on frequency-division multiplexing fiber-loop cavity ring-down spectroscopy," Anal. Chem. 83, 2719-2725 (2011).

6. S. Yakunin and J. Heitz, "Microgrinding of lensed fibers by means of a scanning-probe microscope setup," Appl. Opt. 48, 6172-6177 (2009).

7. D. R. Rivera, C. M. Brown, D. G. Ouzounov, W. W. Webb, and C. $\mathrm{Xu}$, "Use of a lensed fiber for a large-field-of-view, highresolution, fiber-scanning microendoscope," Opt. Lett. 37, 881-883 (2012).

8. S. Cabrini, C. Liberale, D. Cojoc, A. Carpentiero, M. Prasciolu, S. Mora, V. Degiorgio, F. De Angelis, and E. Di Fabrizio, "Axicon lens on optical fiber forming optical tweezers, made by focused ion beam milling," Microelectron. Eng. 83, 804-807 (2006)

9. F. Schiappelli, R. Kumar, M. Prasciolu, D. Cojoc, S. Cabrini, M. De Vittorio, G. Visimberga, A. Gerardino, V. Degiorgio, and E. Di Fabrizio, "Efficient fiber-to-waveguide coupling by a lens on the end of the optical fiber fabricated by focused ion beam milling," Microelectron. Eng. 73-74, 397-404 (2004).

10. J. K. Kim, J. Kim, K. Oh, I. B. Sohn, W. Shin, H. Y. Choi, and B. Lee, "Fabrication of micro Fresnel zone plate lens on a modeexpanded hybrid optical fiber using a femtosecond laser ablation system," IEEE Photonics Technol. Lett. 21, 21-23 (2009).

11. Y. L. Yu, L. Lui, H. Tam, and W. Chung, "Fiber-laser-based wavelength-division multiplexed fiber Bragg grating sensor system," IEEE Photonics Technol. Lett. 13, 702-704 (2001).

12. D. Hunger, C. Deutsch, R. J. Barbour, R. J. Warburton, and J. Reichel, "Laser micro-fabrication of concave, low-roughness features in silica," AIP Adv. 2, 012119 (2012).

13. D. M. B. Kunert, T. P. Meyrath, and H. Giessen, "Fabrication of a fiber-based microcavity with spherical concave fiber tips," Appl. Phys. B 98, 707-710 (2010).

14. M. Mayeh and F. Farahi, "Tailoring Gaussian laser beam shape through controlled etching of single-mode and multimode fibers: simulation and experimental studies," IEEE Sens. J. 12, 168-173 (2012).

15. J. Mervis, A. H. Bloom, G. Bravo, L. Mills, F. Zarinetchi, M. Prentiss, and S. P. Smith, "Aligning and attaching a lens to an optical fiber using light pressure force," Opt. Lett. 18, 325-327 (1993)

16. M. Mirkhalaf, V. M. Murukeshan, S. B. Tor, V. K. Shinoj, and K. Sathiyamoorthy, "Characteristics of stand-alone microlenses in fiber-based fluorescence imaging applications," Rev. Sci. Instrum. 82, 043110 (2011).

17. A. Mori, T. Horiuchi, M. Mizumachi, S. Seino, T. Nakagawa, and K. Suzuki, "Formation of micro lens by laser polymerization," Electron. Commun. Jpn. 95, 59-67 (2012).

18. W. R. Cox, T. Chen, D. Ussery, D. J. Hayes, J. A. Tatum, and D. L. MacFarlane, "Microjetted lenslet triplet fibers," Opt. Commun. 123, 492-496 (1996).

19. D. M. Hartmann, D. J. Reiley, and S. C. Esener, "Microlenses self-aligned to optical fibers fabricated using the hydrophobic effect," IEEE Photonics Technol. Lett. 13, 1088-1090 (2001).

20. C. A. Brackett, "Efficiency of coupling light from stripegeometry GaAs lasers into multimode optical fibers," J. Appl. Phys. 45, 2636-2637 (1974).

21. H. Omrani, J. A. Barnes, A. E. Dudelzak, H.-P. Loock, and H. Waechter, "Fluorescence excitation-emission matrix (EEM) spectroscopy and cavity ring-down (CRD) absorption spectroscopy of oil-contaminated jet fuel using fiber-optic probes," Analyst 137, 2782-2790 (2012).

22. D. Munzke, J. Saunders, H. Omrani, O. Reich, and H.-P. Loock, "Modeling of fiber-optic fluorescence probes for strongly absorbing samples," Appl. Opt. 51, 6343-6351 (2012).

23. G. He and F. W. Cuomo, "A light-intensity function suitable for multimode fiberoptic sensors," J. Lightwave Technol. 9, 545-551 (1991). 